\section{$\underset{\substack{\text { hommes } \\ \text { \& migrations }}}{ }$}

\section{Hommes \& migrations}

Revue française de référence sur les dynamiques

migratoires

$1313 \mid 2016$

1983, le tournant médiatique

\title{
La marche pour l'égalité des droits et contre le racisme
}

Une tentative de dé-monstration?

Piero-D. Galloro

\section{(2) OpenEdition}

Journals

Édition électronique

URL : http://journals.openedition.org/hommesmigrations/3554

DOI : $10.4000 /$ hommesmigrations.3554

ISSN : 2262-3353

Éditeur

Musée national de l'histoire de l'immigration

Édition imprimée

Date de publication : 1 janvier 2016

Pagination : 19-26

ISBN : 978-2-919040-34-6

ISSN : 1142-852X

Référence électronique

Piero-D. Galloro, «La marche pour l'égalité des droits et contre le racisme », Hommes \& migrations [En ligne], 1313 | 2016, mis en ligne le 01 janvier 2019, consulté le 16 mars 2020. URL : http://

journals.openedition.org/hommesmigrations/3554; DOI : https://doi.org/10.4000/

hommesmigrations.3554 


\section{LA MARCHE POUR L'ÉGALITÉ DES DROITS ET CONTRE LE RACISME UNE TENTATIVE DE DÉ-MONSTRATION ?}

par PIERO-D. GALLORO, maître de conférences, HDR, en sociologie, université de Lorraine, Laboratoire lorrain de sciences sociales (2L2S)

Durant I'automne 1983, en allant à pied de Marseille à Paris, les acteurs de cette marche historique ont tenté de redonner des couleurs au visage d'une France blafarde, en pleine crise économique, gagnée par les démons du racisme, enflammée par une vague d'émeutes. Démarche pacifiste, cette action visait autant à exiger la reconnaissance de la place des enfants d'immigrés au sein de la société française qu'à dénoncer les clivages et la violence qui la traversent. Marcher le long des routes de France et donner la chasse à ses monstres.

Le 15 octobre 1983 débute la Marche pour l'égalité des droits et contre le racisme, événement central de l'histoire de l'immigration en France. Central parce que cette manifestation se voulait fondatrice d'une nouvelle conscience républicaine ${ }^{1}$ et s'inscrit dans le contexte des premières années de crise économique, de situation postcoloniale ${ }^{2}$ et de tensions liées aux luttes pour la reconnaissance ${ }^{3}$. Central également parce qu'il consacre l'irruption sur la scène politique française de jeunes, en grande partie des nationaux, dont les parents sont nés dans l'ancien empire colonial français ${ }^{4} .1983$ restera marquée par la démarche de ces héritiers ${ }^{5}$, qui, face aux exactions subies, ont voulu révéler leur existence sociale par la non-violence. Cette volonté de désinvisibilisation trouvera son point d'acmé le 3 décembre suivant dans un rassemblement place de la Bastille, salué par un : «Bonjour à la France de toutes les couleurs » clamé par l'un des principaux initiateurs devant des dizaines de milliers de personnes rassemblées pour l'occasion. Derrière la spontanéité de cet appel universaliste, il convient de comprendre que les marcheurs de 1983 s'inscrivent dans les transformations sociales perceptibles au sein de la société française et entendent également se montrer comme les acteurs des changements sociaux à venir. 


\section{La visibilité croissante des flambées urbaines}

Le 21 mars 1983, une perquisition à la cité Monmousseau-Herriot dans le quartier des Minguettes à Vénissieux dans le Rhône dégénère et des jeunes décident d'entamer une grève de la faim pour protester contre des poursuites judiciaires et dénoncer un sentiment de harcèlement de la part des forces de l'ordre. Les jeunes du quartier vont également, en réaction, créer une association, SOS Avenir Minguettes, dont le président, Toumi Djaija, sera blessé par un policier quelques jours plus tard. L'idée d'une marche pacifiste s'impose alors sur la base du modèle étatsunien de la lutte des Noirs pour leurs droits civiques en lien avec l'idée de nonviolence inspirée par Gandhi Même sila région et l'esprit des paysans du LarIyonnaise està l'initiative zac (que certains marcheurs des contestations, ont rencontrés) et des contesc'est de Marseille que partira

la Marche le 15 octobre 1983 en direction de Paris, après

l'attentat revendiqué par

le groupe Charles Martel dans cette ville et qui a coûté la vie à un enfant de 11 ans. nant des années 1970-1980, des flambées urbaines de plus en plus visibles et médiatisées ${ }^{6}$ mettant en scène l'exclusion d'une certaine jeunesse ${ }^{7}$ et qui dépassent le cadre de l'Hexagone $e^{8}$. De manière générale, d'un problème local, les événements de la région lyonnaise prendront une ampleur nationale. Dans un premier temps, de 1981 à 1983, peu de journaux s'y intéressent de manière suivie. Les rodéos font quatre fois la Une du Progrès de Lyon qui leur consacre quelques articles dans huit éditions. Après les rodéos de 1981, seuls la presse quotidienne régionale et Le Figaro traitent du sujet. Le Figaro évoque de manière laconique, dans son édition du 23 juillet 1981, les événements du 21 en les qualifiant de "chauds ", tandis que Le Monde publie deux articles, le 14 et le 23 juillet 1981, sur la question. En 1983, les manchettes de la presse et la télévision décrivent les cités lyonnaises comme des zones urbaines dangereuses et de non-droit, alors que des images tournées sur le vif par une agence locale, IM'média, créée en juin 1983, tentent de relativiser les propos des autres médias 9 .

Même si la région lyonnaise est à l'initiative des contestations, c'est de Marseille que partira la Marche le 15 octobre 1983 en direction de Paris, après l'attentat revendiqué par le groupe Charles Martel dans cette ville et qui a coûté la vie à un enfant de 11 ans. À mesure que les quelques dizaines de marcheurs évoluent à travers la France, d'Aix-enProvence à Lyon en passant par Avignon, Orange et Vienne, des marches parallèles se mettent en place dans les localités où les marcheurs ne passent pas, comme à Toulouse, Nantes, Rennes, Le Havre ou Saint-Nazaire. Des comités d'accueil se développent pour coordonner les aspects logistiques et donner une visibilité au mouvement, tandis que des appels sont lancés vers la classe politique et les personnalités avec le soutien d'organismes comme la Cimade. $\mathrm{Si}$, au départ, seule la députée socialiste et ancienne maire de Dreux est présente, c'est à Strasbourg que la reconnaissance s'affirme de manière importante avec l'apparition aux côtés des marcheurs de la ministre des Affaires sociales, Georgina Dufoix, qui servira de relais politique.

Elle débouchera par un accueil de quelques marcheurs par le président de la République François Mitterrand en dénonciation des violences racistes. Celles-ci sont, chez les marcheurs, de deux ordres, celui des brutalités policières et celui des conditions de vie des habitants, dont font partie les jeunes marcheurs. Ce mouvement pacifiste s'inscrit à la convergence de plusieurs facteurs sociaux. 


\section{La Marche de 1983 dans une société en mutation}

Tout d'abord, la désindustrialisation débutée au cours des années 1960-1970 devient effective avec la crise pétrolière et, malgré l'arrivée de la gauche au pouvoir en 1981, 1983 sonne le glas de l'engagement de l'État dans nombre de secteurs économiques. Cette tendance s'accompagne du déclin du mouvement ouvrier, de sa représentativité et de sa mobilisation dans bien des secteurs de la vie sociale notamment dans les quartiers où vivent les ouvriers ${ }^{10}$. Ces derniers sont largement issus des migrations, de moins en moins en provenance des pays limitrophes que des anciennes colonies, en particulier d'Afrique du Nord et subsaharienne. À l'image de potentiel se substitue celle de l'immigration comme problème social lié à la montée du chômage et à la dualisation de la société ${ }^{11}$, entre ceux qui disposent des ressources dans la société de consommation et ceux qui en sont exclus ou qui craignent de l'être ${ }^{12}$.

De plus, en 1983, les violences sont, en France, indissociables du contexte particulier de l'espace urbain à travers l'image des HLM et de la banlieue chantés par Renaud en 1980 et par Karim Kacel en 1982, ou la violence dénoncée par Rock Against Police. Si le terme existe depuis le XII ${ }^{e}$ siècle dans la langue française pour désigner l'espace en marge d'une ville, c'est au cours des années 1980 qu'il prendra une connotation spécifique en lien avec d'autres mots tels qu'abandon et bannissement, avec lesquels il partage une même étymologie et contre lesquels s'insurgent les jeunes qui y habitent. En France, ces nouveaux territoires hors des anciens centres historiques ont pris le relais des faubourgs, ces lieux en périphérie mal bâtis et mal habités ${ }^{13}$.
Les Trente Glorieuses verront se multiplier des immeubles à forte densité d'habitants, construits en tours et barres, qui vont devenir des espaces de relégation privilégiés pour les populations issues de l'ancien empire colonial. Après la crise de 1973, les enfants de ces familles installées seront en première ligne de la nouvelle scène contestataire pour dénoncer l'absence de lieux de sociabilité $^{14}$ et l'impression d'abandon des indésirables comme autant « d'assignations à résidence en fonction des revenus $»^{15}$. Avec la dualisation de la société française, une gentrification des centres urbains a De plus, en 1983, les violences sont, en France, indissociables du contexte particulier de l'espace urbain à travers I'image des HLM et de la banlieue chantés par Renaud en 1980 et par Karim Kacel en 1982, ou la violence dénoncée par Rock Against Police. conduit à l'abandon des logements de type HLM au profit de quartiers plus résidentiels par les catégories sociales les moins défavorisées, contribuant à ajouter à l'exclusion sociale une exclusion spatiale. Enfin, le sentiment de déclin d'une France de moins en moins visible dans les affaires internationales ${ }^{16}$, l'influence des décisions européennes, la poussée culturelle anglo-saxonne ${ }^{17}$ et le traumatisme des décolonisations, notamment après l'indépendance de l’Algérie, contribuent à faire émerger les questions identitaires et la peur d'une présence étrangère délétère pour lidentité française.

\section{Effets de l'ethnicisation des rapports sociaux et de la relégation spatiale}

Au cœur des quartiers déclassés, les tensions s'exacerbent au point de devenir visibles à l'échelle nationale avec l'embrasement des Minguettes en juillet 1981 dans le quartier de Monmousseau, mais également à Rillieux-la-Pape ou à Saint-Fons.

10. Alain Touraine, Michel Wieviorka, François Dubet, Le Mouvement ouvrier, Pairs, Fayard, 1984. 11. Alain Ehrenberg, La Société du malaise, Paris, Odile Jacob, 2010. 12. Vincent De Gaulejac, Frédéric Blondel, Isabel Taboada-Leonetti, La Lutte des places, Paris, Desclée de Brouwer, 2015. 13. Alain Faure, "Un faubourg, des banlieues, ou la déclinaison du rejet ", in Genèses, vol. 2, n51, 2003, pp. 48-69. 14. L'Humanité, 4 août 1981. 15. Journal officiel, débats parlementaires, Assemblée nationale, compte rendu intégral, séance du 12 novembre 1981, Loi de finances pour 1982, urbanisme et logement, intervention de Jacqueline Osselin, p. 3551. 16. Luc Pinhas, "La francophonie, le français, son génie et son déclin ", in Documents pour l'histoire du français langue étrangère et seconde, $\mathrm{n}^{\circ}$ 40-41, 2008. 17. Nathalie Dupont, "Le cinéma américain : un impérialisme culturel ? ", in Revue LISA/LISA e-journal, vol. V, n ${ }^{\circ}$, 2007, pp. 111-132. 
Marche pour l'Égalité et contre le racisme, départ le 15 octobre 1983 à Marseille, en présence de la député et ex-maire de Dreux, Françoise Gaspard.

(c) PHoto PierRe CIOT / AGENCE IM'MÉDIA.

Très rapidement, dans les mois qui suivent les premières images télévisées des rodéos jusqu'aux blessures infligées à Toumi Djaidja, des incidents similaires sont signalés en région parisienne, à Avignon ou dans l'est de la France, comme à Mulhouse ou à Metz, à Marseille ou dans le quartier du Pont Rompu de Tourcoing ${ }^{18}$. La Marche de 1983 met en évidence les conditions de vie des individus et des groupes notamment à partir du paramètre architectural omniprésent dans le paysage. Les troubles de l'année 1983 et l'initiative des marcheurs incitent les autorités à réagir et constituent de ce point de vue une étape essentielle dans le développement de la politique de la Ville depuis les années 1970 avec, en 1983, la mise en place, entre juillet et septembre, de la mission Banlieue $89^{19}$, dans le prolongement des rapports Schwartz ${ }^{20}$, Bonnemaison $^{21}$ et Dubedout ${ }^{22}$. Les choix urbains des décennies précédentes seront pointés du doigt autant par les jeunes que par les autorités, dans la continuité des critiques du rapport Bonnemaison pour qui les bandes et les groupes sont un produit urbain ${ }^{23}$. "Les incidents qui émaillent tristement les nuits de certaines banlieues ne sont qu'une des conséquences de la

18. Bernard Alidières, Géopolitique de l'insécurité et du Front national, Paris, Armand Colin, 2006. 19. "À propos de Banlieue 89: entretien avec l'architecte Roland Castro ", in Cahiers d'histoire. Revue d'histoire critique, n¹09, 2009, pp. 95-97. 20. Bertrand Schwartz, "L'insertion professionnelle et sociale des jeunes. Rapport au Premier ministre ", Paris, La Documentation française, 1981. 21. Gilbert Bonnemaison, «Face à la délinquance : prévention, répression, solidarité. Rapport au Premier ministre, Commission des maires sur la sécurité ", Paris, La Documentation française, 1982. 22. Hubert Dubedout, "Ensemble refaire la ville. Rapport au Premier ministre du président de la Commission nationale pour le développement social des quartiers ", Paris, La Documentation française, 1983. 23. Gilbert Bonnemaison, op. cit., p. 33. 
ségrégation engendrée par la politique d'affairisme et de désengagement ${ }^{24}$. "L'idée que le «béton des grands ensemble est criminogène ${ }^{25}$ » contribuera au développement de dispositifs spécifiques, en particulier les premiers contrats de plan État-régions pour le développement social des quartiers.

Il convient également de prendre conscience qu'au-delà de l'aspect architectural et urbain, l'espace conditionne l'ethnicisation des relations entre les populations qui l'habitent. La plupart des cités impliquées depuis des années et notamment en 1983 - (telle la cité de la Grapinière de Vaulx-enVelin où se sont déroulés les premiers conflits) ont été construites dès la fin de la guerre d’Algérie pour accueillir les supplétifs de l'armée française, tandis que la cité Simion à Villeurbanne, à l'instar des immeubles de la ZUP des Minguettes de Vénissieux, accueillent le même profil de locataires. Les journaux télévisés ne se priveront pas de rappeler la présence de plusieurs dizaines de nationalités différentes ${ }^{26}$.

Dans cette cohabitation entre rapatriés d'Algérie, harkis, primo-arrivants nord-africains, populations locales et Domiens, les multiples interactions sociales entre ceux qui se posent comme établis et les nouveaux venus provoquent, chez les habitants les plus anciens des quartiers, un sentiment d'envahissement face aux derniers arrivés perçus comme des intrus. Tout cela engendre des logiques d'exclusion ${ }^{27}$, dont l'étude des relations quotidiennes suffit à montrer le caractère clivant ${ }^{28}$. Elles se traduisent par la montée des votes de l'extrême droite qui vient, en 1983, de remporter la municipalité de Dreux sur la base d'un rejet de l'immigration, et par des amalgames que d'autres partis politiques cautionnent et reproduisent en consacrant la réduction de la question migratoire en France à une approche en termes de "problèmes ». Ainsi en est-il de la confusion entre les étrangers et les jeunes des banlieues (pour la plupart nés français en France), dont une partie de la classe politique dénonce "la prolifération et les chiffres impressionnants ${ }^{29}$ ". Cette catégorisation se traduit également par une série de violences et de faits d'armes qui s'ajoutent à une longue liste égrenant, depuis le début des années 1970, les exactions sur des jeunes d'origine nord-africaine ${ }^{30}$. La blessure de Toumi Djaidja va servir de point de départ à la Marche et le meurtre de Habib Grimzi dans le Bordeaux-Vinimille en sera le point d'orgue. Le sentiment d'impunité dont jouissent les auteurs de violences achève d'exaspérer les tensions entre jeunes et policiers, révélant une volonté de prendre à contre-pied la spirale mortifère par une action pacifiste.

Plus que cette mise en visibilité des conditions de vie, c'est la prise de conscience d'une différenciation entre populations qui émerge. Les jeunes s'insurgent contre les catégoLes regards portés au cours des années 1970 et 1980 sur ces enfants de migrants nord-africains, et notamment de parents algériens, restent imprégnés des représentations élaborées au cours des années 1950 sur leurs propres parents, au moment de la guerre d'Algérie. risations construites à partir d'éléments biologiques ou culturels établis par la suite comme étant " naturellement naturelles ${ }^{31}$ ", et qui servent à alimenter à leur tour les convictions de dangerosité de ces quartiers et a fortiori de leurs habitants ${ }^{32}$. La violence est analysée par nombre de contemporains de la Marche comme étant le fait de voyous, de voleurs de véhicules ${ }^{33}$, par la faute de qui les quartiers sont devenus dangereux ${ }^{34}$ et qui sévertuent à créer les fameuses zones noires, véritables lieux de non-droit, dénoncées alors par la press $e^{35}$. Les regards portés au cours des années 1970 et 1980 sur ces enfants de migrants

24. Journal officiel, débats parlementaires, Assemblée nationale, compte rendu intégral, séance du 12 novembre 1981 , op. cit., p. 3549. 25. Paris Match, 26 août 1983. 26. Laurent Massardier, Antenne 2, Midi 2, 14 septembre 1981, 2’42. 27. Norbert Elias, John Scotson, Logiques de l'exclusion, Paris, Fayard, 1997. 28. Michelle Zancarini-Fournel, "Généalogie des rébellions urbaines en temps de crise (1971-1981)", in Vingtième Siècle - Revue d'histoire, vol. 4, n84, 2004, pp. 119-127. 29. Jacques Chirac, Le Monde, 15 juillet 1983. 30. Fabien Jobard, Bavures policières? La force publique et ses usages, Paris, La Découverte, 2002. 31. Abdelmalek Sayad, "Naturels et naturalisés ", in Actes de la recherche en sciences sociales, vol. 99, Septembre 1993, pp. 26-35 32. Voir à ce sujet les travaux de Raymond Boudon, L'Art de se persuader des idées douteuses, fragiles ou fausses, Paris, Fayard, 199o, et les travaux spécifiques sur l'autoréalisation des prophéties de Robert Merton, Éléments de théorie et de méthode sociologique, Paris, Colin, 1998. 33. Le Figaro, 3 août 1981. 34. Le Figaro, 10 juillet 1981. 35. Le Figaro, 23 juillet 1981. 
nord-africains, et notamment de parents algériens, restent imprégnés des représentations élaborées au cours des années 1950 sur leurs propres parents, au moment de la guerre d'Algérie. Est véhiculée l'image de violence des Nord-Africains dans la presse extrémiste française qui n'hésite pas à clamer que «les Arabes adorent la guerre ${ }^{36}$ » ou " avec les Arabes, une seule politique est possible, celle de la trique et du coup de pied au cul ${ }^{37}$ ! ». Ce type de discours est omniprésent dans les périodes de tensions sociales, lorsque les médias contribuent à faire émerger un profil type des responsables du chaos en élaborant la figure du jeune de bande ${ }^{38}$, dangereux car violent, généralement de sexe masculin ${ }^{39}$ et surtout d'origine maghrébine ${ }^{40}$.

\section{La marche pacifiste, une stratégie de retournement du stigmate}

C'est donc dans une posture de retournement du stigmate que les jeunes - qui sont toujours plus nombreux à défier les forces de l'ordre - choisissent une ligne pacifiste. Ils sont soutenus par le curé Christian Delorme et le pasteur Jean Costi ${ }^{41}$. Les marcheurs clament des slogans tels que "Rengainez on arrive. La chasse est fermée ", "Égalité des droits, justice pour tous ", "Raciste... moi ? Jamais » ou "Différents mais égaux »" et même "Couscous chez Mitterrand ».

Nous pouvons y lire un double positionnement. D'une part, le refus des violences comme révélateur d'une égalité de traitement. L'idée de violence, par sa multiplicité reste mouvante, insaisissable et changeante, d'autant plus qu'en fonction des lieux et des temporalités elle peut désigner des réalités différentes ${ }^{43}$. La première s'attache à la manifestation concrète de ce que Bessette qualifiait de crime contre les individus ${ }^{44}$, tout en pouvant être considérée d'un point de vue plus immatériel, en s'intéressant aux rapports sociaux à travers les structures ${ }^{45}$ ou les relations symboliques ${ }^{46}$. La première forme de violence, marquée dans les gestes et les corps, est facilement identifiable par ses formes directes que sont les coups et les agressions; les crimes dits " racistes » des années précédant la Marche en sont des exemples concrets. Toutefois, les autres modalités n'en sont pas moins, en dépit de leur immatérialité apparente, des réalités sociales qu'il convient de révéler à travers l'analyse de leurs mécanismes. La violence se manifeste alors par des faits et des actions qui portent atteinte aux personnes et à leurs biens et illustre l'imposition d'un rapport de domination ${ }^{47}$ porteur de sens. Ainsi, l'analyse des discours et des actes sur les jeunes héritiers des migrations coloniales rend lisibles des pratiques de relégation. Les contrôles au faciès, la violence ordinaire ${ }^{48}$ peuvent être vus comme des formes de violence invisible propres à imposer une différenciation sociale et politique à partir de marqueurs biologiques ou cognitifs ${ }^{49}$.

D'autre part, en brandissant la non-violence comme étendard, les jeunes des Minguettes s'évertuent à entrer dans une logique de démonstration, dans le sens premier du terme, «monstre » (celui qui est dans la monstration, celui que l'on montre, désigne ou met à l'index). Parler de "monstres » à propos des jeunes de 1983, c'est donc s'interroger sur la construction sociale qui touche au fantastique, au mythe et à la légende, et qui affecte des individus et des groupes au sein d'une société. La définition du monstre ne peut se contenter de le limiter à son seul aspect physiologique comme continuent à le faire la plupart des dictionnaires.

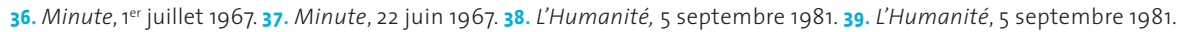
40. Le Figaro, 23 juillet 1981. 41. Le Figaro, 23 mars 1983. 42. Michel Kokoreff, "L’imaginaire social de la Marche de 1983", in Africultures, "La Marche en héritage - L'héritage culturel de la Marche pour l'égalité et contre le racisme (1983-2013)", vol. 1, $n^{\circ}$ 97, 2014, pp. 28-42. 43. Jean-Claude Chesnais, Histoire de la violence en Occident de 1800 à nos jours, Paris, Robert Laffont, 1981. 44. Jean-Michel Bessette, Sociologie du crime, Paris, PUF, 1982. 45. Johan Galtung, « Violence, peace and peace research ", in Journal of Peace Research, vol. 6, n³, 1969, pp. 167-191. 46. Pierre Bourdieu, Réponses, pour une anthropologie réflexive, Paris, Seuil, pp. 146-147; Slavoj Zyzek, La violenza invisibile, Milano, Rizzoli, 2007 [1992]. 47. Yves Michaud, La Violence, Paris, PUF, coll. "Que sais-je? ", n`2251, 2012. 48. Andrea Réa-Maryse Tripier, Sociologie de l'immigration, La Découverte, 2008, p. 74 49. Frédéric Barth, Ethnic Groups and Boundaries : the Social Organisation of Culture Différence, Bergen/Oslo, Universitetsforlaget, London, George Allen and Uwin, 1969. 
rence $e^{52}$ ». Or le monstre voit sa monstruosité définie dans l'ambiguité puisqu'il appartient au genre humain comme un «individu singulier qui n'entre pas vraiment dans la catégorie à laquelle il appartient ${ }^{53}$ ». Dès lors, plusieurs voies s'offrent à son évolution. Il peut rester monstre qui vit comme un homme mais n'y ressemble pas. Il peut aussi accéder au statut d'homme.

\section{La banalité du commun ou les désirs déçus des marcheurs}

En 1983, les jeunes des banlieues ont cherché à être appréhendés dans un rapport de banalité avec les autres nationaux tout en revendiquant leur appartenance aux mondes des migrations. Dans le sens étymologique, le banal, c'est ce qui est « commun ». D'abord dans son premier sens de trivial, d'ordinaire. Un second sens, plus riche, renvoie selon les dictionnaires à l'idée de participation col-

(C) GÉNÉRIQUUES

Il convient d'accepter sa dimension doxique, de l'ordre du sens commun, qui élargit la notion purement tératologique de monstre à toute difformité pouvant susciter le jugement $t^{50}$. Le monstre n'est pas à chercher dans l'exceptionnel, l'accidentel, mais dans la quotidienneté, dans cette connaissance ordinaire qui révèle les mécanismes les plus fins de cette capacité qu'ont les individus à effectuer des tractations épistémiques nécessaires pour agir en commun, et en particulier à catégoriser, abstraire, généraliser ou anticiper ${ }^{51}$. À ce propos, Robert Bogdan rappelle que l'étude des monstres «(...) permet de mieux comprendre certaines pratiques sociales, de retracer l'évolution du concept d'anormalité et de théoriser le regard que nous portons sur la diffélective - tout le monde peut en profiter -, comme dans les expressions "le commun des mortels", "le droit commun », «le dénominateur commun », etc. Cette participation collective se double d'une idée d'action menée ensemble, conjointement, comme dans faire " cause commune ", " avoir quelque chose en commun", "l'intérêt commun ". Cette définition se rattache à l'origine latine «communis », qui définit les personnes qui supportent ensemble (cum) les charges (munus). Pour parvenir à l'agir en commun - qui donne le sentiment d'égalité -, il est nécessaire de montrer que le niveau ordinaire n'est pas dépassé mais qu'au contraire il existe une conformité au plus grand nombre. Or cette conformité n'a rien de naturel puisqu'elle est le produit de l'action humaine. En cela, la banalité apparait comme le contenu d'une certaine forme de regard porté sur le réel, comme un produit 
d'opérations qu'il semble naturel à l'homme d'accomplir. Les initiateurs de la Marche ont eu donc recours à une mise en scène visant à établir un équilibre complexe entre la spécificité des enfants issus des migrations et leur permanence en tant que citoyens à part entière, entre êtres d'exception et gens du commun. Pour Bergson, c'est le langage qui est le vecteur de la banalité par sa puissance évocatrice. La parole, comme intention expressive et pratique, devient une substitution du symbole à la réalité ${ }^{54}$. Le langage, tels les slogans, les banderoles, donne à voir une certaine réalité, et c'est par elle que se crée l'idée de communion entre les individus, malgré les différences flagrantes de statut, de position sociale ou de niveau de richesse. "La Marche, c'était d'autres gens, pas que des Maghrébins, des filles, des garçons, de tout âge, des curés, des moines, des élèves, des clandestins (...). C'était une conjugaison de choses qui a fait qu'un groupe de personnes qui avaient entre 17 et un peu plus de 50 ans (...). C'était pas une histoire de jeunes Arabes, cétait l'histoire d'un groupe de personnes qui disaient "Halte au feu". C'était l'un des premiers slogans de la Marche pour l'égalité et contre le racisme ${ }^{55}$.»

En étant accueillis avec enthousiasme le 3 décembre 1983 par des milliers de personnes, puis à l'Élysée par le représentant de l'État français, les marcheurs ont pu mettre en scène cette idée d'égalité banale qui idéalement régit les affaires publiques. Le spectacle offert (du latin spectaculum, qui lui-même provient de spectare, regarder, orienter le regard) a pris alors tout son sens. Celui d'une véritable exposition à l'attention publique qu'une partie de la population française refusait la posture de démonstration imposée jusque-là au détriment d'une jeunesse liée par son ascendance à la question migratoire et coloniale sur un air de Douce France orientalisé qu'entonnera le groupe Carte de Séjour quelques mois plus tard. Le désarroi d'une telle proposition est d'autant plus perceptible dans la société française de 1983 qu'il est relayé, dans les médias, par l'incapacité de nommer ce mouvement pour ce qu'il est, celui de jeunes qui cherchent à montrer qu'ils ne sont pas dans la double absence dénoncée par Abdelmalek Sayad ${ }^{56}$, mais qu'ils revendiquent leur double présence dans leur héritage migratoire et leur appartenance nationale. L'emploi, par ces mêmes médias, de vocables tels que " deuxième génération d'immigrés " pour désigner les marcheurs et la réduction de la Marche pour l'égalité des droits et contre le racisme en Marche des " Beurs $^{57}$ », va prendre le contre-pied des intentions originelles et ouvrir la voie à une ré-invisibilisation durable des aspirations égalitaristes et pacifistes du mouvement.

54. Lucien Jerphagnon, "Entre la solitude et la banalité : philosophie bergsonienne du banal ", in Revue de métaphysique et de morale, ${ }^{\circ} 3,1962$, pp. 322-329. 55. Intervention de Farid L'Haoua, porte-parole et photographe de la Marche de 1983, in Piero-D. Galloro, Ahmed Boubeker (dir.), "L'héritage de la Marche pour l'égalité et contre le racisme de 1983 ou les épreuves d'une affirmation publique de la diversité culturelle ", table ronde 2 , Médiatisation, espace public et dimension multiculturelle, colloque de Metz, 12 et 13 décembre 2013. 56. Abdelmalek Sayad, La Double Absence: des illusions de l'émigré aux souffrances de l'immigré, Paris, Seuil, 1999 57. "La Marche des jeunes Franco-Arabes "pour l'égalité" traverse Paris - Paris sur 'Beur'», in Libération, 3 décembre 1983 